\title{
Cosmological Alfvén waves in the recent CMB data, and the observational bound on the primordial vector perturbation
}

\author{
Jaiseung $\mathrm{Kim}^{1}$ and Pavel Naselsky ${ }^{1}$ \\ ${ }^{1}$ Niels Bohr Institute, Blegdamsvej 17, DK-2100 Copenhagen, Denmark \\ E-mail: jkim@nbi.dk
}

\begin{abstract}
In the presence of the primordial magnetic field, initial vector (vorticity) perturbations produce cosmological Alfvén waves and leave imprints on cosmic microwave background (CMB) temperature and polarization anisotropy.

We have investigated imprints of cosmological Alfvén waves in CMB anisotropy. For data constraints, we have used the power spectrum of the recent CMB observations, and correlations estimated from WMAP Internal Linear Combination (ILC) maps. Our analysis shows $3 \sigma$ evidence of cosmological Alfvén waves. Using the $3 \sigma$ limit from our analysis and the Alfvén velocity limit from the total energy density constraint, we impose a lower bound on the amplitude of primordial vector perturbation: $4 \times 10^{-12}$ at $k_{0}=0.002 / \mathrm{Mpc}$.
\end{abstract}

PACS numbers: 95.85.Sz, 98.70.Vc, 98.80.Cq, 98.80.Es, 98.80.-k

\section{Introduction}

There are strong observational evidences on the existence of large-scale magnetic fields, whose strength is on the order of micro Gauss [1, 2, 3, 4. These magnetic fields are believed to be seeded by a small Primordial Magnetic Field (PMF) [5, 6, 7, 8]. Primordial Magnetic Field may be considered to consist of a homogeneous and inhomogeneous (stochastic) magnetic fields. In this paper, we focus on the effect of Primordial Magnetic Field on CMB anisotropy associated with vector perturbations. Primordial vector perturbation, whose source includes topological defects and inhomogeneous primordial magnetic fields [9, 10, 11, decays rapidly with the expansion of the Universe, and therefore, does not leave observable signature on CMB anisotropy [12, 13]. However, primordial vector perturbation in the presence of a homogeneous primordial magnetic field induces Alfvén waves in the cosmic plasma, which may leave observable imprints on CMB anisotropy via a Doppler effect and an integrated Sachs-Wolfe effect [14, 15, 16, 17, 18, 19]. To be specific, cosmological Alfvén waves create correlations between $a_{l m}, a_{l \pm \Delta l, m \pm \Delta m}$, where $\Delta l=0,1,2$ and $\Delta m=0,1,2$ [20]. Over the past several years, there have been great success in measurement of Cosmic Microwave Background (CMB) anisotropy by ground and satellite observations [21, 22, 23, 24, 25, 26, 27, 28, 
and observational imprints of cosmological Alfvén waves in CMB anisotropy have been studied by several authors [18, 20, 29, 30, 31]. By investigating the effect on power spectrum and signature correlations in the recent CMB data, we attempt to constrain cosmological Alfvén waves, and also impose a lower bound on the primordial vector perturbation.

The outline of this paper is as follows. In Section 2, we briefly review the effect of cosmological Alfvén waves on CMB anisotropy. In Section 3, we discuss statistical properties of $\mathrm{CMB}$ in the presence of the Alfvén waves. In Section 4, we build the statistics sensitive to the imprints of Alfvén waves. In Section 5, we analyze the recent CMB data and present the result. In Section 6, we make summary and conclusion. In Appendix A, we discuss the effect of incomplete sky coverage on the analysis of Alfvén wave imprints.

\section{The effect of Alfvén waves on CMB anisotropy}

According to linearized Einstein equations [32, vector perturbation decays rapidly with the expansion of the Universe, and therefore, does not leave observable signature on CMB anisotropy, unless the initial values of vector perturbation are unusually high [12, 13. In the presence of a homogeneous magnetic field, vector perturbation induces Alfvén waves in cosmic plasma, and leaves observable imprints on CMB anisotropy via a Doppler effect and an integrated Sachs-Wolfe effect [18]. Durrer, Kahniashvili and Yates [18] (hereafter, DKY) showed that cosmological Alfvén waves generate the fractional CMB anisotropy for a Fourier mode $\mathbf{k}$ :

$$
\frac{\delta T}{T_{0}}(\hat{\mathbf{n}}, \mathbf{k}) \approx \hat{\mathbf{n}} \cdot \boldsymbol{\Omega}\left(\mathbf{k}, \eta_{d e c}\right)=\hat{\mathbf{n}} \cdot \boldsymbol{\Omega}_{\mathbf{0}} v_{A} k \eta_{d e c} \hat{\mathbf{B}} \cdot \hat{\mathbf{k}}
$$

where $\hat{\mathbf{n}}$ and $\hat{\mathbf{B}}$ denote sky direction and a homogeneous magnetic field direction respectively, $\boldsymbol{\Omega}\left(\mathbf{k}, \eta_{\text {dec }}\right)$ is the Gauge invariant linear combination associated with vector perturbations, $\eta_{d e c}$ denotes the conformal time at the moment of baryon-photon decoupling, $v_{A}$ is Alfvén wave velocity and $T_{0}$ is the CMB monopole temperature $2.725[\mathrm{~K}][33$. DKY assumed that vector perturbations are initially created by some

random process and have the following statistical properties over an ensemble of universes:

$$
\left\langle\Omega_{0}^{i}(\mathbf{k}) \Omega_{0}^{j}(\mathbf{k})\right\rangle=\left(\delta_{i j}-\hat{k}_{i} \hat{k}_{j}\right) P(k),
$$

$P(k)$, which is the power spectrum, is assumed to follow a simple power law:

$$
P(k)=A_{v} \frac{k^{n}}{k_{0}^{n+3}},
$$

where $k_{0}$ is a pivot wavenumber and we set it to $0.002 / \mathrm{Mpc}$, which is equal to the WMAP team's pivot wavenumber for scalar perturbation [34]. Possible sources of primordial vector perturbation include inhomogeneous stochastic primordial magnetic fields and topological defects [9, 10, 11]. 


\section{CMB statistical properties}

The temperature anisotropy $\delta T(\theta, \phi)$ is conveniently decomposed in terms of spherical harmonics $Y_{l m}(\theta, \phi)$ :

$$
\delta T(\theta, \phi)=\sum_{l m} a_{l m} Y_{l m}(\theta, \phi),
$$

where $a_{l m}$ are the coefficients of decomposition, and $\theta$ and $\phi$ are a polar and an azimuthal angle. Kahniashvili, Lavrelashvili and Ratra [20] (hereafter KLR) showed that the CMB anisotropy in the presence of cosmological Alfvén waves has the following statistical properties:

$$
\begin{aligned}
& \left\langle a_{l m}^{*} a_{l m}\right\rangle=C_{l}^{0}+\left(3 \cos ^{2} \theta_{B}-1\right) \frac{l(l+1)}{(2 l-1)(2 l+3)} \\
& \times\left\{\frac{l(l+1)+\left(l^{2}+l-3\right) \cos ^{2} \theta_{B}}{3 \cos ^{2} \theta_{B}-1}-m^{2}\left[1-\frac{3}{l(l+1)}\right]\right\} I_{d}^{l, l} \\
& \left\langle a_{l m}^{*} a_{l, m+1}\right\rangle=-\sin 2 \theta_{B} \exp \left[-i \phi_{B}\right] I_{d}^{l, l} d_{l l}^{m, m+1}, \\
& \left\langle a_{l m}^{*} a_{l, m+2}\right\rangle=-\frac{1}{2} \sin ^{2} \theta_{B} \exp \left[-2 i \phi_{B}\right] I_{d}^{l, l} d_{l l}^{m, m+2}, \\
& \left\langle a_{l, m}^{*} a_{l+2, m}\right\rangle=-\left(3 \cos ^{2} \theta_{B}-1\right) I_{d}^{l, l+2} d_{l, l+2}^{m, m}, \\
& \left\langle a_{l, m}^{*} a_{l+2, m+1}\right\rangle=\sin 2 \theta_{B} \exp \left[-i \phi_{B}\right] I_{d}^{l, l+2} d_{l, l+2}^{m, m+1}, \\
& \left\langle a_{l, m}^{*} a_{l+2, m-1}\right\rangle=\sin 2 \theta_{B} \exp \left[i \phi_{B}\right] I_{d}^{l, l+2} d_{l, l+2}^{m, m-1}, \\
& \left\langle a_{l, m}^{*} a_{l+2, m+2}\right\rangle=-\frac{1}{2} \sin ^{2} \theta_{B} \exp \left[-2 i \phi_{B}\right] I_{d}^{l, l+2} d_{l, l+2}^{m, m+2}, \\
& \left\langle a_{l, m}^{*} a_{l+2, m-2}\right\rangle=-\frac{1}{2} \sin ^{2} \theta_{B} \exp \left[2 i \phi_{B}\right] I_{d}^{l, l+2} d_{l, l+2}^{m, m-2}
\end{aligned}
$$

where $C_{l}^{0}$ is the power spectrum in the absence of Alfvén waves, $\theta_{B}$ and $\phi_{B}$ are the spherical coordinate of a PMF direction $\hat{\mathbf{B}}$, and $d_{l l^{\prime}}^{m, m^{\prime}}\left(|m| \leq l,\left|m^{\prime}\right| \leq l^{\prime}\right)$ are

$$
\begin{aligned}
d_{l, l}^{m, m+1} & =\frac{l^{2}+l-3}{(2 l-1)(2 l+3)}\left(m+\frac{1}{2}\right) \sqrt{(l-m)(l+m+1)}, \\
d_{l, l}^{m, m+2} & =\frac{l^{2}+l-3}{(2 l-1)(2 l+3)} \sqrt{(l-m)(l-m-1)(l+m+1)(l+m+2)}, \\
d_{l, l+2}^{m, m} & =\frac{(l+3) l}{2(2 l+3) \sqrt{(2 l+1)(2 l+5)}} \sqrt{\left((l+1)^{2}-m^{2}\right)(l-m+2)(l+m+2)}, \\
d_{l, l+2}^{m, m+1} & =\frac{(l+3) l}{2(2 l+3) \sqrt{(2 l+1)(2 l+5)}} \sqrt{T\left((l+1)^{2}-m^{2}\right)(l+m+2)(l+m+3)}, \\
d_{l, l+2}^{m, m-1} & =\frac{(l+3) l}{2(2 l+3) \sqrt{(2 l+1)(2 l+5)}} \sqrt{\left((l+1)^{2}-m^{2}\right)(l-m+2)(l-m+3)},
\end{aligned}
$$




$$
\begin{aligned}
& d_{l, l+2}^{m, m+2}=\frac{(l+3) l}{2(2 l+3) \sqrt{(2 l+1)(2 l+5)}} \sqrt{\frac{(l+m+4) !}{(l+m) !}}, \\
& d_{l, l+2}^{m, m-2}=\frac{(l+3) l}{2(2 l+3) \sqrt{(2 l+1)(2 l+5)}} \sqrt{\frac{(l-m+4) !}{(l-m) !}},
\end{aligned}
$$

and

$$
\begin{aligned}
I_{d}^{l l^{\prime}} & =\frac{2 T_{0}^{2}}{\pi} \int k^{2}\left(P_{0} \frac{k^{n_{v}}}{k_{0}^{n_{v}+3}}\right) \exp \left(-2 \frac{k^{2}}{k_{D}^{2}}\right) v_{A}^{2}\left(\frac{\eta_{d e c}}{\eta_{0}}\right)^{2} j_{l}\left(k \eta_{0}\right) j_{l}^{\prime}\left(k \eta_{0}\right) d k, \\
& =\frac{2 T_{0}^{2}}{\pi} \frac{P_{0} v_{A}^{2}}{\left(k_{0} \eta_{0}\right)^{n_{v}+3}}\left(\frac{\eta_{d e c}}{\eta_{0}}\right)^{2} \int x^{n_{v}+2} \exp \left(-\frac{2 x^{2}}{\left(k_{D} \eta_{0}\right)^{2}}\right) j_{l}(x) j_{l}^{\prime}(x) d x .
\end{aligned}
$$

$\eta_{0}$ is the present conformal time, and $v_{A}$ is the Alfvén velocity, which is given by

$$
v_{A}^{2}=\frac{B_{0}^{2}}{4 \pi\left(\rho_{r}+p_{r}\right)}, \quad v_{A} \sim 4 \times 10^{-4} \frac{B_{0}}{10^{-9} \text { Gauss }},
$$

where $B_{0}$ is the magnitude of a homogeneous PMF, and $\rho_{r}$ and $p_{r}$ are the density and the pressure of photons [18]. $k_{D}$ denotes the comoving wavenumber of the dissipation scale, due to photon viscosity and given by $\sim 10 / \eta_{\text {dec }}[18$. While DKY and KLR have obtained analytic results by neglecting the damping factor $\exp \left(-2 k^{2} / k_{D}^{2}\right)$, we have computed Eq. 13 numerically. The damping effect is getting significant on multipoles $l \gtrsim 500[18$.

Primordial magnetic fields affect the expansion dynamics of the Universe, because of anisotropic pressures associated with primordial magnetic fields [35]. Considering the shear anisotropy due to primordial magnetic fields and the observed magnitude of CMB anisotropy $\left(\sim 10^{-5}\right)$, Barrow et al. (1997) have derived an upper limit $B_{0}<2.27 \times 10^{-9} h / 75$ Gauss on a homogeneous primordial magnetic field [35]. Using this upper limit and Eq. 14, DKY showed $B_{0} \lesssim 10^{-8}$ Gauss and $v_{A} \lesssim 10^{-3}$ [18].

It is worth to note that correlations are invariant under the parity inversion of a PMF direction $\hat{\mathbf{B}}$. In other words, $\left.\left(\theta_{B}, \phi_{B}\right) \rightarrow\left(\pi-\theta_{B}, \phi_{B}+\pi\right)\right)$ does not affect Eq. 6, 7, 8, 9, 10, 11 and 12. note that it is the degeneracy of correlation, not that there should be two physical directions.

\section{Estimators}

In order to constrain cosmological Alfvén waves, we construct the following statistics:

$$
\begin{aligned}
C_{l} & =(2 l+1)^{-1} \sum_{m} a_{l m}^{*} a_{l m}, \\
D_{l}^{1} & =l^{-1} \sum_{m \geq 0} a_{l m}^{*} a_{l, m+1}, \\
D_{l}^{2} & =(2 l-1)^{-1} \sum_{m} a_{l m}^{*} a_{l, m+2},
\end{aligned}
$$


Cosmological Alfvén waves and the observational bound on the vector perturbation

$$
\begin{aligned}
D_{l}^{3} & =(2 l+1)^{-1} \sum_{m} a_{l, m}^{*} a_{l+2, m}, \\
D_{l}^{4} & =(2 l)^{-1} \sum_{m} a_{l, m}^{*} a_{l+2, m+1}, \\
D_{l}^{5} & =(2 l-1)^{-1} \sum_{m} a_{l, m}^{*} a_{l+2, m+2} .
\end{aligned}
$$

Because of the reality condition $a_{l-m}=(-1)^{m} a_{l m}{ }^{*}, \sum_{m=-2 l} a_{l m}^{*} a_{l, m+1}$ is always zero. Therefore, we defined $D_{l}^{1}$ to sum only terms of $m \geq 0$. There also exist the following indentities because of the reality condition:

$$
\begin{aligned}
\sum_{m} a_{l, m}^{*} a_{l+2, m+1} & =-\left[\sum_{m} a_{l, m}^{*} a_{l+2, m-1}\right]^{*}, \\
\sum_{m} a_{l, m}^{*} a_{l+2, m+2} & =\left[\sum_{m} a_{l, m}^{*} a_{l+2, m-2}\right]^{*} .
\end{aligned}
$$

Therefore, $\sum_{m} a_{l, m}^{*} a_{l+2, m-1}$ and $\sum_{m} a_{l, m}^{*} a_{l+2, m-2}$ are redundant and excluded from consideration. Note that the statistic used in [18, 29] is equivalent to Eq. 18, Using Eq. 5, 6, 7, 8, 9 and 11, we may easily show that the expectation values of $C_{l}$ and $D_{l}^{i}$ are

$$
\begin{aligned}
& \bar{C}_{l}=C_{l}^{0}+\frac{l(l+1)}{3} I_{d}^{l, l}, \\
& \bar{D}_{l}^{1}=-\sin 2 \theta_{B} \exp \left[-i \phi_{B}\right] \frac{I_{d}^{l, l}}{l} \sum_{m \geq 0} d_{l l}^{m, m+1}, \\
& \bar{D}_{l}^{2}=-\frac{1}{2} \sin ^{2} \theta_{B} \exp \left[-2 i \phi_{B}\right] \frac{I_{d}^{l, l}}{2 l-1} \sum_{m} d_{l l}^{m, m+2}, \\
& \bar{D}_{l}^{3}=-\left(3 \cos ^{2} \theta_{B}-1\right) \frac{I_{d}^{l, l+2}}{2 l+1} \sum_{m} d_{l, l+2}^{m, m}, \\
& \bar{D}_{l}^{4}=\sin 2 \theta_{B} \exp \left[-i \phi_{B}\right] \frac{I_{d}^{l, l+2}}{2 l} \sum_{m} d_{l, l+2}^{m, m+1}, \\
& \bar{D}_{l}^{5}=-\frac{1}{2} \sin ^{2} \theta_{B} \exp \left[-2 i \phi_{B}\right] \frac{I_{d}^{l, l+2}}{2 l-1} \sum_{m} d_{l, l+2}^{m, m+2} .
\end{aligned}
$$

We may also show that the variance of $D_{l}^{i}$ is

$$
\begin{aligned}
& \operatorname{Var}\left(C_{l}\right) \approx 2 /(2 l+1)\left(C_{l}^{0}+N_{l}\right)^{2}, \\
& \operatorname{Var}\left(D_{l}^{1}\right) \approx l^{-1}\left(C_{l}^{0}+N_{l}\right)^{2}, \\
& \operatorname{Var}\left(D_{l}^{2}\right) \approx(2 l-1)^{-1}\left(C_{l}^{0}+N_{l}\right)^{2}, \\
& \operatorname{Var}\left(D_{l}^{3}\right) \approx(2 l+1)^{-1}\left(C_{l}^{0}+N_{l}\right)\left(C_{l+2}^{0}+N_{l+2}\right), \\
& \operatorname{Var}\left(D_{l}^{4}\right) \approx(2 l)^{-1}\left(C_{l}^{0}+N_{l}\right)\left(C_{l+2}^{0}+N_{l+2}\right), \\
& \operatorname{Var}\left(D_{l}^{5}\right) \approx(2 l-1)^{-1}\left(C_{l}^{0}+N_{l}\right)\left(C_{l+2}^{0}+N_{l+2}\right),
\end{aligned}
$$

where $N_{l}$ is noise power spectrum, and we have neglected correlations between distinct spherical harmonic modes (i.e. $\left\langle a_{l m}^{*} a_{l^{\prime} m^{\prime}}\right\rangle \approx C_{l}^{0} \delta_{l l^{\prime}} \delta_{m m^{\prime}}$ ). 
Though the underlying distribution for primordial vector perturbations is not necessarily Gaussian, the distribution function of $D_{l}^{i}-\bar{D}_{l}^{i}$ tends to Gaussian by the central limit theorem [36]. Hence, the likelihood function of $\bar{D}_{l}^{i}$, given the data $D_{l}^{i}$, is

$$
\mathcal{L}\left(\bar{D}_{l}^{i}\left(\lambda_{\alpha}\right) \mid D_{l}^{i}\right)=\frac{1}{(2 \pi)^{\frac{N}{2}}|\mathbf{M}|^{\frac{1}{2}}} \exp \left[-\frac{1}{2}\left(\mathbf{D}-\overline{\mathbf{D}}\left(\lambda_{\alpha}\right)\right)^{\dagger} \mathbf{M}^{-1}\left(\mathbf{D}-\overline{\mathbf{D}}\left(\lambda_{\alpha}\right)\right)\right]
$$

$\mathbf{M}$ is a $5 l_{\max } \times 5 l_{\max }$ nearly diagonal matrix, whose diagonal elements correspond to the variances of $D_{l}^{1}, \ldots, D_{l}^{5}: \mathbf{M} \approx \operatorname{diag}\left(\operatorname{Var}\left(D_{l_{\min }}^{1}\right), \ldots, \operatorname{Var}\left(D_{l_{\max }}^{5}\right)\right) . N$ is the data vector size (i.e. $5 l_{\max }$ ), and $\lambda_{\alpha}$ are the parameters associated with Alfvén waves : $\lambda_{\alpha} \in\left\{A_{v} v_{A}^{2}, n_{v}, \theta_{B}, \phi_{B}\right\}$. For low $l$, the deviation of $D_{l}^{i}-\bar{D}_{l}^{i}$ from Gaussian distribution is getting non-negligible. However, it does not affect the best-fit values, but only the error bars, since $\mathbf{M}$ is nearly diagonal, and thereofore the best-fit parameters are, in fact, determined by minimizing $\chi^{2}$. Since $\left\langle\left(C_{l}-\bar{C}_{l}\right)\left(D_{l}^{i}-\bar{D}_{l}^{i}\right)\right\rangle$ is negligible in comparison to the variance of $C_{l}$ or $D_{l}^{i}$, we may construct a full likelihood function as follows:

$$
\mathcal{L}\left(\bar{C}_{l} \mid C_{l}\right) \times \mathcal{L}\left(\bar{D}_{l}^{i}\left(\lambda_{\alpha}\right) \mid D_{l}^{i}\right),
$$

where $\mathcal{L}\left(\bar{C}_{l} \mid C_{l}\right)$ is the likelihood function associated with CMB power spectra, whose approximate and optimal expression is found in [37. Note that cosmological Alfvén waves affect $\mathrm{CMB}$ power spectrum as well, and hence Eq. 23 should be used for $\bar{C}_{l}$ in estimating $\mathcal{L}\left(\bar{C}_{l} \mid C_{l}\right)$. Since CMB power spectra and correlations (i.e. $\bar{C}_{l}$ and $\bar{D}_{l}^{i}$ ) possess non-trivial dependence on cosmological parameters (i.e. standard parameters + Alfvén wave parameters), we will resort to Markov Chains Montel Carlo (MCMC) likelihood analysis [37, 38]. The discussion on MCMC analysis and dataset used will be give in the next section.

\section{Analysis of the recent CMB observation data}

We have investigated the effect of the WMAP team's KQ75 and KQ85 mask on correlations (i.e. $D_{l}^{i}$ ), and found that significant amount of fictitious correlations are produced by the masks (refer to Appendix A for details). Therefore, we find sky maps, which require a Galactic mask, are not suitable for our analysis. Therefore, we have restricted our correlation estimation to whole sky maps obtained by variants of Internal Linear Combination (ILC) method: The WMAP team's ILC map (WILC), Harmonic ILC (HILC) and Needlet ILC (NILC) [39, 40, 41, 42, 43. In Fig. 1, we show the temperature power spectra of the ILC maps with that of the WMAP concordance model [34, 23]. Noting that the power spectra of ILC maps are in good agreement with the model up to $l \leq 200$, we have computed $D_{l}^{i}$ up to $l \leq 200$. We have also made the analysis with $D_{l \leq 250}^{i}$ for HILC, and $D_{l \leq 250}^{i}$ and $D_{l \leq 300}^{i}$ respectively for NILC. We have obtained the consistent results with the $D_{l \leq 200}^{i}$ analysis. The confidence intervals are smaller, but the best-fit values turn out to be similar. From now on, all the results are from the $D_{l \leq 200}^{i}$ analysis. 


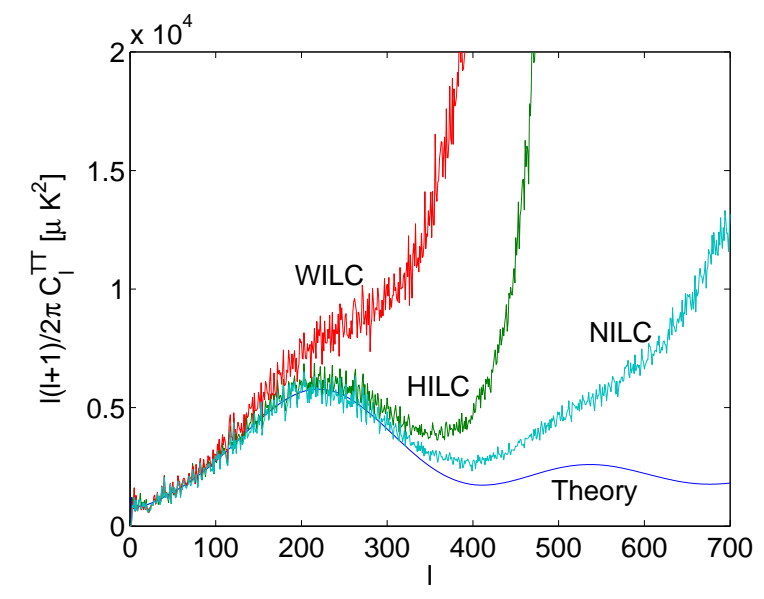

Figure 1. the power spectra of ILC maps and the WMAP concordance model

Table 1. cosmological parameters of $\Lambda \mathrm{CDM}+\left(A_{v} v_{A}^{2}, n_{v}, \theta_{B}, \phi_{B}\right)$ constrained by power spectra (WMAP5YR + ACBAR + QUaD) + correlation (ILC)

\begin{tabular}{cccc}
\hline parameter & WILC & HILC & NILC \\
\hline$\Omega_{b} h^{2}$ & $0.023_{-0.002}^{+0.002}$ & $0.024_{-0.003}^{+0.002}$ & $0.024_{-0.003}^{+0.002}$ \\
$\Omega_{c} h^{2}$ & $0.109_{-0.024}^{+0.023}$ & $0.104_{-0.018}^{+0.03}$ & $0.108_{-0.024}^{+0.025}$ \\
$\tau$ & $0.089_{-0.04}^{+0.085}$ & $0.09_{-0.043}^{+0.082}$ & $0.089_{-0.04}^{+0.08}$ \\
$n_{s}$ & $1.015_{-0.139}^{+0.262}$ & $1.028_{-0.123}^{+0.242}$ & $1.018_{-0.13}^{+0.251}$ \\
$d n_{s} / d \ln k$ & $-0.008_{-0.095}^{+0.068}$ & $-0.008_{-0.095}^{+0.052}$ & $-0.009_{-0.108}^{+0.066}$ \\
$\log \left[10^{10} A_{s}\right]$ & $3.031_{-0.27}^{+0.17}$ & $2.975_{-0.234}^{+0.228}$ & $3.026_{-0.321}^{+0.188}$ \\
$A_{\mathrm{sz}}$ & $1.63_{-1.63}^{+0.37}$ & $1.87_{-1.867}^{+0.133}$ & $1.57_{-1.57}^{+0.43}$ \\
$H_{0}[\mathrm{~km} / \mathrm{s} / \mathrm{Mpc}]$ & $74.37_{-10.58}^{+13.65}$ & $76.62_{-13.19}^{+10.19}$ & $74.46_{-10.98}^{+13.55}$ \\
$10^{9} A_{v} v_{A}^{2}$ & $3.13_{-2.42}^{+5.66}$ & $4.16_{-3.27}^{+4.99}$ & $3.87_{-3.11}^{+5.28}$ \\
$n_{v}$ & $-4.35_{-0.7}^{+0.58}$ & $-4.45_{-0.71}^{+0.6}$ & $-4.49_{-0.7}^{+0.73}$ \\
$\theta_{B}^{+}$ & $15.7_{-15.7}^{\circ+25}$ & $1.6_{-1.6}^{\circ+31.7}$ & $11.7_{-11.7}^{\circ+27.7}$ \\
$\phi_{B}^{+}$ & $112_{-111.8}^{\circ+247.9}$ & $113.2_{-113.1}^{\circ+246.6}$ & $119_{-119}^{\circ+240.9}$ \\
$\theta_{B}^{-}$ & $164.3_{-25}^{\circ+15.7}$ & $178.4_{-31.7}^{\circ+1.6}$ & $168.3_{-2.7}^{\circ+11.7}$ \\
$\phi_{B}^{-}$ & $292_{-111.8}^{\circ+247.9}$ & $293.2_{-113.1}^{\circ+246.6}$ & $299_{-119}^{\circ+240.9}$ \\
\hline
\end{tabular}

By making small modifications to the CosmoMC package [38], we have included the parameter $\lambda_{\alpha} \in\left\{A_{v} v_{A}^{2}, n_{v}, \theta_{B}, \phi_{B}\right\}$, and computed the likelihood function given by Eq. 36. Note that the power spectrum as well as correlations depend on $A_{v} v_{A}^{2}$ and $n_{v}$ (see Eq. 23). For data constraint, we have used the CMB power spectra of the recent CMB observations (WMAP5YR + ACBAR + QUaD) [21, 22, 24, 25, 26, 27, 28, and the correlations estimated from ILC maps. In Table 1, we summarize $1 \sigma$ constraint and the best-fit values. As discussed in Sec. 4, our estimators are insensitive to the parity of a PMF direction. Hence, we quote two best-fit PMF directions, which are equally likely. In Fig. 2, we show the marginalized likelihood (solid lines) and mean likelihood (dotted lines) of $\left\{A_{v} v_{A}^{2}, n_{v}, \theta_{B}, \phi_{B}\right\}$. In Fig. 3, we show likelihood in the plane of $A_{v} v_{A}^{2}$ 

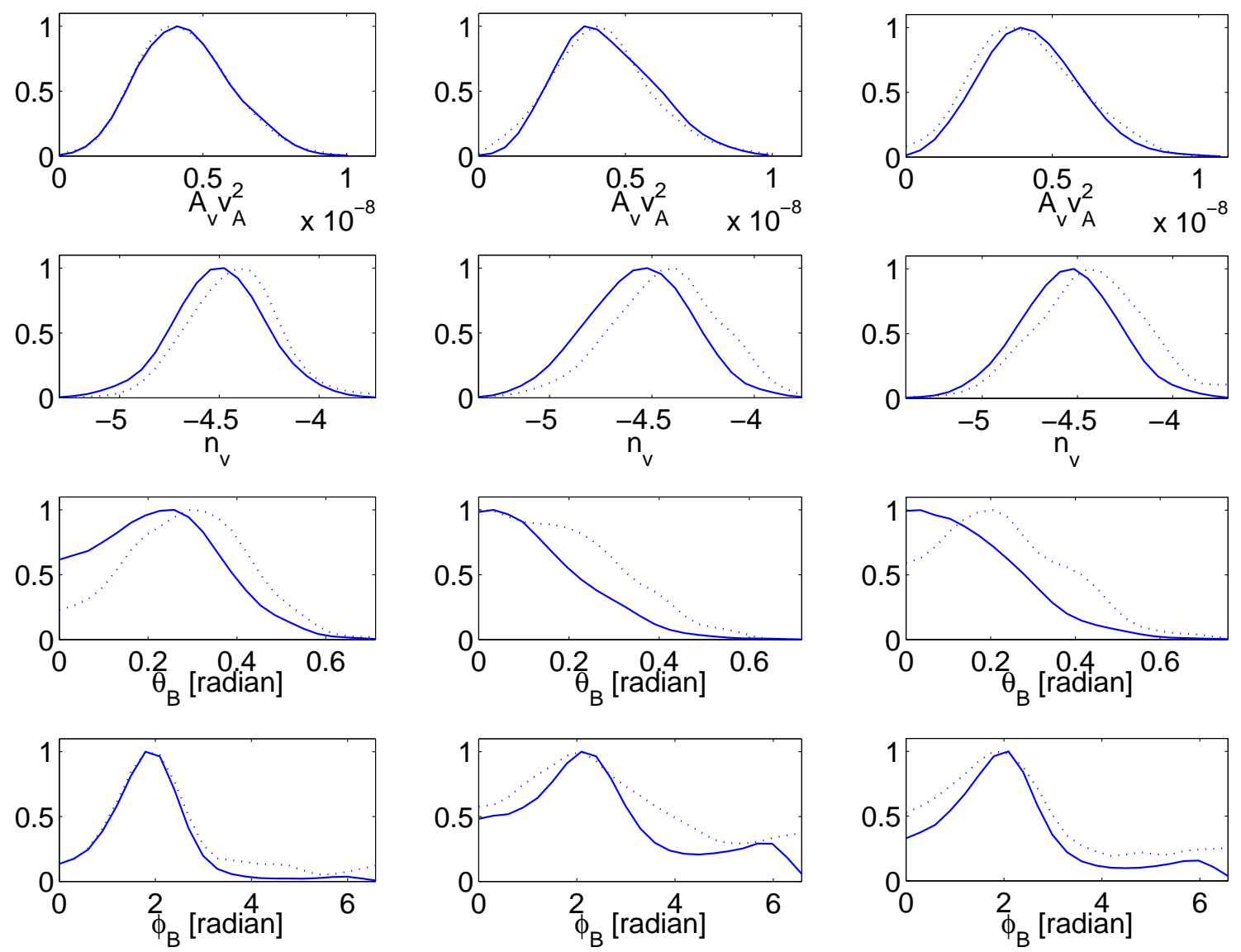

Figure 2. Likelihood of Alfvén wave parameters: WILC, HILC and NILC (from the left to the right), normalized to its peak.

versus $n_{v}$, which are highly correlated Alfvén wave parameters. We find there exists little correlation between Alfvén wave parameters and $\Lambda$ CDM parameters, except for the scalar perturbation amplitude $A_{s}$. In Fig. 3, we show marginalized likelihood in the plane of $A_{v} v_{A}^{2}$ versus $\log \left[10^{10} A_{s}\right]$. Marginalized likelihood of PMF direction is shown in Fig. 4. Note that a pair of directions associated by $\left(\theta_{B}, \phi_{B}\right) \leftrightarrow\left(\pi-\theta_{B}, \phi_{B}+\pi\right)$ possess equal likelihood. As shown in Table 1 and Fig. 2, 3, 4, The result from various ILC maps are similar with each other. Since each ILC map contains distinct residual foregrounds and noise, consistency among the analysis with various ILC maps indicates that our results are affected insignificantly by residual foregrounds and noise in ILC maps.

For good exploration on tails of the parameter $A_{v} v_{A}^{2}$, we have also set CosmoMC to check convergence for confidence intervals of $A_{v} v_{A}^{2}$. In Table 2, we show $3 \sigma$ constraints on $A_{v} v_{A}^{2}$. Using our $3 \sigma$ limit on $A_{v} v_{A}^{2}$ and the upper limit on $v_{A}$ (refer to Sec. 3), we find $5 \times 10^{-4} \lesssim A_{v}$ (refer to Eq. 3 for the definition of $A_{v}$ ). Taking into account Eq. 

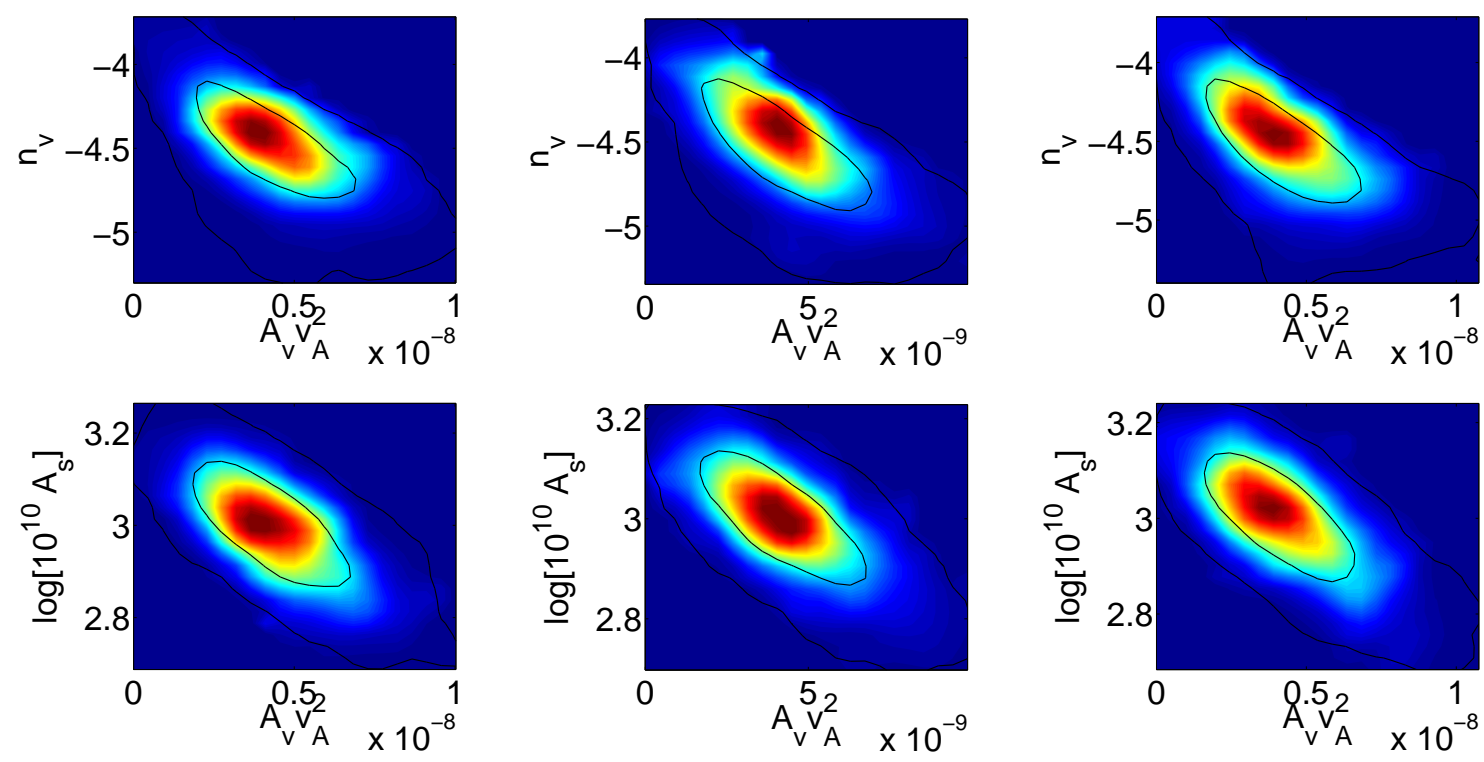

Figure 3. Marginalized likelihood in the plane of $A_{v} v_{A}^{2}$ vs $\left\{n_{v}, \log \left[10^{10} A_{s}\right]\right\}$ : WILC, HILC and NILC (from left to right), solid curves denote $1 \sigma$ and $2 \sigma$ contours.
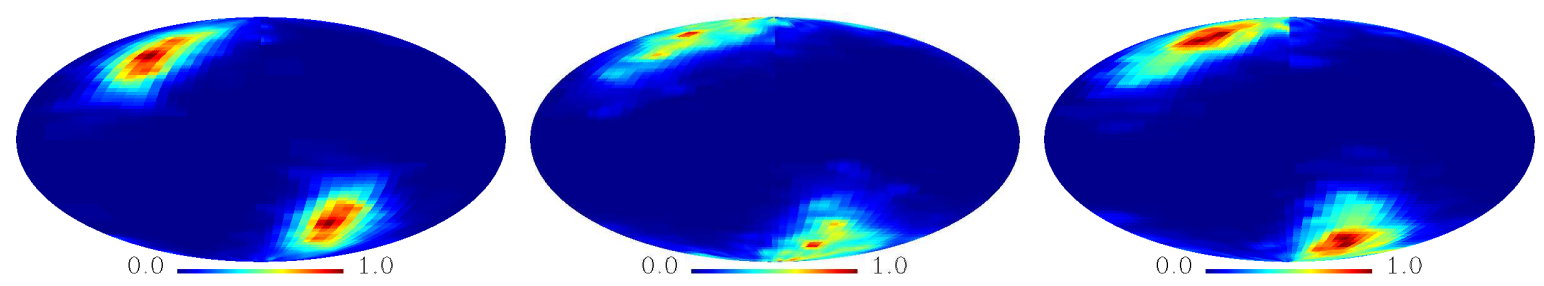

Figure 4. Marginalized likelihood of PMF direction: WILC, HILC, and NILC (from left to right), normalized to its peak

3. we find the amplitude of primordial vector perturbation is equal to $A_{v} k_{0}^{3}$. Hence, we impose the lower bound $4 \times 10^{-12}$ on the amplitude of primordial vector perturbation.

Table 2. $A_{v} v_{A}^{2}$ estimation at $3 \sigma$ confidence level

\begin{tabular}{cc}
\hline Power Spectra + ILC & $3 \sigma$ confidence interval \\
\hline WILC & $5.8 \times 10^{-10}<A_{v} v_{A}^{2}<1.01 \times 10^{-8}$ \\
HILC & $5.73 \times 10^{-10}<A_{v} v_{A}^{2}<0.99 \times 10^{-8}$ \\
NILC & $5.05 \times 10^{-10}<A_{v} v_{A}^{2}<1.09 \times 10^{-8}$ \\
\hline
\end{tabular}

If there was a primordial magnetic field at the recombination epoch or after the Universe was re-ionized, it would induce Faraday rotation in CMB polarization [44, 45]. Forecast on the PLANCK data constraint shows that we will be able to constrain a primordial magnetic field $\sim 2 \times 10^{-9}$ Gauss by investigating Faraday rotation effect in polarization data [45]. However, we have not considered Faraday rotation effect in this 
work, since the signal-to-noise ratio in the currently available CMB polarization data is not high.

\section{Discussion}

We have constrained primordial magnetic field and primordial vector perturbation, by investigating the imprints of cosmological Alfvén waves on CMB anisotropy. We find there is little degeneracy between cosmological Alfvén wave parameters and $\Lambda \mathrm{CDM}$ parameters except for a scalar perturbation amplitude $A_{s}$. The results obtained with various ILC maps are consistent with each other. Therefore, we believe our results have not been affected significantly by residual foregrounds or anisotropic noise in ILC maps. Using our result and the upper bound on $v_{A}$ from the total energy density constraint, we impose a lower bound on the primordial vector perturbation $5 \times 10^{-4} \lesssim A_{v}$ at the pivot scale $k_{0}=0.002 / \mathrm{Mpc}$.

Since Alfvén velocity $v_{A}$ is proportional to the magnitude of a primordial magnetic field, direct constraints on Alfvén velocity $v_{A}$ may be imposed by investigating Faraday rotation effect in CMB polarization data [44, 45. When the data from the PLANCK surveyor [46] are available, we are going to constrain Alfvén waves and Faraday rotation simultaneously, and hence impose a stronger constraint on the primordial vector perturbation.

\section{Acknowledgments}

We thank anonymous referees for helpful comments, which lead to sharpening this work. We thank T. Kahniashvili and G. Lavrelashvili for useful discussions. We also thank J. Delabrouille and his colleagues for making their whole-sky CMB map (NILC) publically available. We acknowledge the use of the Legacy Archive for Background Data Analysis (LAMBDA) and ACBAR, and QUaD data. Our data analysis made the use of HEALPix [47, 48, and the CosmoMC package. This work is supported by FNU grant 272-06-0417, 272-07-0528 and 21-04-0355.

\section{Reference}

[1] A. M. Wolfe, K. M. Lanzetta, and A. L. Oren. Magnetic fields in damped Ly-alpha systems. Astrophys. J., 388:17-22, March 1992.

[2] T. E. Clarke, P. P. Kronberg, and H. Böhringer. A New Radio-X-Ray Probe of Galaxy Cluster Magnetic Fields. Astrophys. J. Lett., 547:L111-L114, February 2001.

[3] L. M. Widrow. Origin of galactic and extragalactic magnetic fields. Reviews of Modern Physics, 74:775-823, 2002.

[4] Y. Xu, P. P. Kronberg, S. Habib, and Q. W. Dufton. A Faraday Rotation Search for Magnetic Fields in Large-scale Structure. Astrophys. J., 637:19-26, January 2006.

[5] T. Vachaspati. Magnetic fields from cosmological phase transitions. Physics Letters B, 265:258261, August 1991.

[6] G. Baym, D. Bödeker, and L. McLerran. Magnetic fields produced by phase transition bubbles in the electroweak phase transition. Phys. Rev. D, 53:662-667, January 1996. 
[7] J. M. Quashnock, A. Loeb, and D. N. Spergel. Magnetic field generation during the cosmological QCD phase transition. Astrophys. J. Lett., 344:L49-L51, September 1989.

[8] B. Cheng and A. V. Olinto. Primordial magnetic fields generated in the quark-hadron transition. Phys. Rev. D, 50:2421-2424, August 1994.

[9] N. Turok, U.-L. Pen, and U. Seljak. Scalar, vector, and tensor contributions to CMB anisotropies from cosmic defects. Phys. Rev. D, 58(2):023506-+, July 1998.

[10] U. Seljak, U.-L. Pen, and N. Turok. Polarization of the Microwave Background in Defect Models. Phys. Rev. Lett., 79:1615-1618, September 1997.

[11] D. Paoletti, F. Finelli, and F. Paci. The full contribution of a stochastic background of magnetic fields to CMB anisotropies. MNRAS in press, November 2008.

[12] Viatcheslav Mukhanov. Physical Foundations of Cosmology. Cambridge University Press, 1st edition, 2005.

[13] Steven Weinberg. Cosmology. Oxford University Press, 1st edition, 2008.

[14] J. Adams, U. H. Danielsson, D. Grasso, and H. Rubinstein. Distortion of the acoustic peaks in the CMBR due to a primordial magnetic field. Physics Letters B, 388:253-258, February 1996.

[15] K. Subramanian and J. D. Barrow. Microwave Background Signals from Tangled Magnetic Fields. Physical Review Letters, 81:3575-3578, October 1998.

[16] K. Subramanian and J. D. Barrow. Small-scale microwave background anisotropies arising from tangled primordial magnetic fields. Mon. Not. R. Astron. Soc., 335:L57-L61, September 2002.

[17] K. Subramanian, T. R. Seshadri, and J. D. Barrow. Small-scale cosmic microwave background polarization anisotropies due to tangled primordial magnetic fields. Mon. Not. R. Astron. Soc., 344:L31-L35, September 2003.

[18] R. Durrer, T. Kahniashvili, and A. Yates. Microwave background anisotropies from Alfvén waves. Phys. Rev. D, 58:123004, 1998.

[19] K. Subramanian and J. D. Barrow. Magnetohydrodynamics in the early universe and the damping of nonlinear Alfvén waves. Phys. Rev. D, 58(8):083502-+, October 1998.

[20] T. Kahniashvili, G. Lavrelashvili, and B. Ratra. CMB temperature anisotropy from broken spatial isotropy due to a homogeneous cosmological magnetic field. Phys. Rev. D, 78(6):063012, September 2008.

[21] G. Hinshaw and et al. Five-year wilkinson microwave anisotropy probe (WMAP) observations: Data processing, sky maps, and basic results. 2008. arXiv:0803.0732.

[22] M. R. Nolta and et al. Five-year Wilkinson Microwave Anisotropy probe (WMAP) observations: Angular power spectra. submitted to Astrophys.J.Suppl., 2008. arXiv:0803.0593.

[23] J. Dunkley, E. Komatsu, M. R. Nolta, D. N. Spergel, D. Larson, G. Hinshaw, L. Page, C. L. Bennett, B. Gold, N. Jarosik, J. L. Weiland, M. Halpern, R. S. Hill, A. Kogut, M. Limon, S. S. Meyer, G. S. Tucker, E. Wollack, and E. L. Wright. Five-Year Wilkinson Microwave Anisotropy Probe Observations: Likelihoods and Parameters from the WMAP Data. Astrophys.J.Suppl., 180:306-329, February 2009.

[24] M. C. Runyan, P. A. R. Ade, R. S. Bhatia, J. J. Bock, M. D. Daub, J. H. Goldstein, C. V. Haynes, W. L. Holzapfel, C. L. Kuo, A. E. Lange, J. Leong, M. Lueker, M. Newcomb, J. B. Peterson, J. Ruhl, G. Sirbi, E. Torbet, C. Tucker, A. D. Turner, and D. Woolsey. Acbar: The arcminute cosmology bolometer array receiver. Astrophys.J.Suppl., 149:265, 2003.

[25] C. L. Reichardt, P. A. R. Ade, J. J. Bock, J. R. Bond, J. A. Brevik, C. R. Contaldi, M. D. Daub, J. T. Dempsey, J. H. Goldstein, W. L. Holzapfel, C. L. Kuo, A. E. Lange, M. Lueker, M. Newcomb, J. B. Peterson, J. Ruhl, M. C. Runyan, and Z. Staniszewski. High resolution cmb power spectrum from the complete ACBAR data set. To be submitted to Astrophys. J., 2008. arXiv:0801.1491.

[26] P. Ade, J. Bock, M. Bowden, M. L. Brown, G. Cahill, J. E. Carlstrom, P. G. Castro, S. Church, T. Culverhouse, R. Friedman, K. Ganga, W. K. Gear, J. Hinderks, J. Kovac, A. E. Lange, E. Leitch, S. J. Melhuish, J. A. Murphy, A. Orlando, R. Schwarz, C. O'Sullivan, L. Piccirillo, C. Pryke, N. Rajguru, B. Rusholme, A. N. Taylor, K. L. Thompson, E. Y. S. Wu, and M. Zemcov. 
First season quad cmb temperature and polarization power spectra. Astrophys. J., 674:22, 2008.

[27] QUaD collaboration: C. Pryke, P. Ade, J. Bock, M. Bowden, M. L. Brown, G. Cahill, P. G. Castro, S. Church, T. Culverhouse, R. Friedman, K. Ganga, W. K. Gear, S. Gupta, J. Hinderks, J. Kovac, A. E. Lange, E. Leitch, S. J. Melhuish, Y. Memari, J. A. Murphy, A. Orlando, R. Schwarz, C. O’Sullivan, L. Piccirillo, N. Rajguru, B. Rusholme, A. N. Taylor, K. L. Thompson, A. H. Turner, E. Y. S. Wu, and M. Zemcov. Second and third season QUaD CMB temperature and polarization power spectra. ArXiv e-prints, May 2008.

[28] QUaD collaboration: J. Hinderks, P. Ade, J. Bock, M. Bowden, M. L. Brown, G. Cahill, J. E. Carlstrom, P. G. Castro, S. Church, T. Culverhouse, R. Friedman, K. Ganga, W. K. Gear, S. Gupta, J. Harris, V. Haynes, J. Kovac, E. Kirby, A. E. Lange, E. Leitch, O. E. Mallie, S. Melhuish, A. Murphy, A. Orlando, R. Schwarz, C. O' Sullivan, L. Piccirillo, C. Pryke, N. Rajguru, B. Rusholme, A. N. Taylor, K. L. Thompson, C. Tucker, E. Y. S. Wu, and M. Zemcov. Quad: A high-resolution cosmic microwave background polarimeter. ArXiv eprints, May 2008.

[29] G. Chen, P. Mukherjee, T. Kahniashvili, B. Ratra, and Y. Wang. Looking for Cosmological Alfvén Waves in Wilkinson Microwave Anisotropy Probe Data. Astrophys. J., 611:655-659, August 2004.

[30] P. D. Naselsky, L.-Y. Chiang, P. Olesen, and O. V. Verkhodanov. Primordial Magnetic Field and Non-Gaussianity of the One-Year Wilkinson Microwave Anisotropy Probe Data. Astrophys. J., 615:45-54, November 2004.

[31] A. Bernui and W. S. Hipólito-Ricaldi. Can a primordial magnetic field originate large-scale anomalies in WMAP data? Mon. Not. R. Astron. Soc., 389:1453-1460, September 2008.

[32] Sean Carroll. Spacetime and Geometry: An Introduction to General Relativity. Benjamin Cummings, 2003.

[33] D. J. Fixsen, E. S. Cheng, J. M. Gales, J. C. Mather, R. A. Shafer, and E. L. Wright. The cosmic microwave background spectrum from the full COBE FIRAS data set. Astrophys. J., 473:576, 1996.

[34] D. N. Spergel and et al. Wilkinson Microwave Anisotropy probe WMAP three year results: Implications for cosmology. Astrophys.J.Suppl., 170:377, 2007.

[35] J. D. Barrow, P. G. Ferreira, and J. Silk. Constraints on a Primordial Magnetic Field. Physical Review Letters, 78:3610-3613, May 1997.

[36] S. J. Bence (Author) K. F. Riley M. P. Hobson (Author). Mathematical Methods for Physics and Engineering: A Comprehensive Guide. Cambridge University Press, 3rd edition, 2006.

[37] L. Verde, H. V. Peiris, D. N. Spergeland M. Noltaand C. L. Bennettand M. Halpernand G. Hinshawand N. Jarosikand A. Kogutand M. Limon, and S. S. Meyerand L. Pageand G. S. Tuckerand E. Wollackand E. L. Wright. First year wilkinson microwave anisotropy probe (WMAP) observations: Parameter estimation methodology. Astrophys.J.Suppl., 148:195, 2003.

[38] Antony Lewis and Sarah Bridle. Cosmological parameters from CMB and other data: a MonteCarlo approach. Phys. Rev. D, 66:103511, 2002.

[39] G. Hinshaw and et al. Three-year Wilkinson Microwave Anisotropy Probe (WMAP) observations: Temperature analysis. Astrophys.J.Suppl., 170:288, 2007.

[40] B. Gold and et al. Five-year wilkinson microwave anisotropy probe (WMAP) observations: Galactic foreground emission. submitted to Astrophys.J.Suppl., 2008. arXiv:0803.0715.

[41] J. Kim, P. Naselsky, and P. R. Christensen. CMB map derived from the WMAP data through harmonic internal linear combination. Phys. Rev. D, 77(10):103002-+, May 2008.

[42] J. Kim, P. Naselsky, and P. R. Christensen. CMB polarization map derived from the WMAP 5year data through the harmonic internal linear combination method. Phys. Rev. D, 79(2):023003-+, January 2009. http://www.nbi.dk/ jkim/hilc.

[43] J. Delabrouille, J.-F. Cardoso, M. Le Jeune, M. Betoule, G. Fay, and F. Guilloux. A full sky, low foreground, high resolution CMB map from WMAP. submitted to AA, 2008. arXiv:0807.0773.

[44] C. Scóccola, D. Harari, and S. Mollerach. B polarization of the CMB from Faraday rotation. 
Phys. Rev. D, 70(6):063003-+, September 2004.

[45] J. R. Kristiansen and P. G. Ferreira. Constraining primordial magnetic fields with CMB polarization experiments. Phys. Rev. D, 77(12):123004-+, June 2008.

[46] European Space Agency. Planck: The scientific programme (blue book). http://www.rssd.esa.int/SA/PLANCK/docs/Bluebook-ESA-SCI2005. Version 2.

[47] K. M. Gorski, B. D. Wandelt, F. K. Hansen, E. Hivon, and A. J. Banday. The HEALPix primer. astro-ph/9905275, 1999.

[48] K. M. Gorski, E. Hivon, A. J. Banday, B. D. Wandelt, F. K. Hansen, M. Reinecke, and M. Bartelman. HEALPix - a framework for high resolution discretization, and fast analysis of data distributed on the sphere. Astrophys. J., 622:759, 2005. http://healpix.jpl.nasa.gov.

\section{Appendix A. incomplete sky coverage}

Incomplete sky coverage produces fictitious correlations by destroying the orthogonality of spherical harmonics. Spherical harmonic coefficients from partial sky coverage is related to the true ones as follows: $a_{l m}=\sum W_{l l^{\prime} m m^{\prime}} a_{l^{\prime} m^{\prime}}^{\text {true }}$, where $W_{l l^{\prime} m m^{\prime}}=$ $\int d^{2} \hat{\mathbf{n}} W(\hat{\mathbf{n}}) Y_{l m}^{*}(\hat{\mathbf{n}}) Y_{l^{\prime} m^{\prime}}(\hat{\mathbf{n}})$, and $W(\hat{\mathbf{n}})$ is zero in the masked region and one elsewhere. To estimate the amount of leakage, we assume CMB is purely Gaussian and has zero correlation:

$$
\left\langle\left(a_{l m}^{\text {true }}\right)^{*} a_{l^{\prime}, m^{\prime}}^{\text {true }}\right\rangle=C_{l} \delta_{l l^{\prime}} \delta_{m m^{\prime}}
$$

We find the expectation value of $D_{l}^{3}$ of the pure Gaussian CMB is

$$
\begin{aligned}
& \left\langle D_{l}^{3}\right\rangle=\frac{1}{2 l+1} \sum_{m}\left\langle a_{l, m}^{*} a_{l+2, m}\right\rangle \\
& =\frac{1}{2 l+1} \sum_{m} \sum_{l^{\prime} m^{\prime}} C_{l^{\prime}} W_{l l^{\prime} m m^{\prime}}^{*} W_{l+2, l^{\prime} m m^{\prime}} \\
& \approx \frac{C_{l+1}}{2 l+1} \sum_{m} \sum_{l^{\prime} m^{\prime}} \int d^{2} \hat{\mathbf{n}} W^{*}(\hat{\mathbf{n}}) Y_{l m}(\hat{\mathbf{n}}) Y_{l^{\prime} m^{\prime}}^{*}(\hat{\mathbf{n}}) \int d^{2} \hat{\mathbf{n}}^{\prime} W\left(\hat{\mathbf{n}}^{\prime}\right) Y_{l+2 m}^{*}\left(\hat{\mathbf{n}}^{\prime}\right) Y_{l^{\prime} m^{\prime}}\left(\hat{\mathbf{n}}^{\prime}\right) \\
& =\frac{C_{l+1}}{2 l+1} \sum_{m} \int d^{2} \hat{\mathbf{n}} W^{*}(\hat{\mathbf{n}}) Y_{l m}(\hat{\mathbf{n}}) \int d^{2} \hat{\mathbf{n}}^{\prime} W\left(\hat{\mathbf{n}}^{\prime}\right) Y_{l+2 m}^{*}\left(\hat{\mathbf{n}}^{\prime}\right) \delta\left(\hat{\mathbf{n}}-\hat{\mathbf{n}}^{\prime}\right) \\
& =\frac{C_{l+1}}{2 l+1} \sum_{m} \int d^{2} \hat{\mathbf{n}}|W(\hat{\mathbf{n}})|^{2} Y_{l m}(\hat{\mathbf{n}}) Y_{l+2 m}^{*}(\hat{\mathbf{n}}) .
\end{aligned}
$$

In the third equality, we have taken $C_{l^{\prime}}$ out of the summation and equate it to $C_{l+1}$, since $W_{l l^{\prime} m m^{\prime}}^{*} W_{l+2, l^{\prime} m m^{\prime}}$ peaks sharply around $l^{\prime}=l+1$, while $C_{l^{\prime}}$ varies much slowly in comparison to $W_{l l^{\prime} m m^{\prime}}^{*} W_{l+2, l^{\prime} m m^{\prime}}$. In the fourth equality, we have used the identity $\sum_{l^{\prime} m^{\prime}} Y_{l^{\prime} m^{\prime}}(\hat{\mathbf{n}}) Y_{l^{\prime} m^{\prime}}\left(\hat{\mathbf{n}}^{\prime}\right)=\delta\left(\hat{\mathbf{n}}-\hat{\mathbf{n}}^{\prime}\right)$. From Eq. A.1, we see the leakage from Gaussian CMB to $D_{l}^{3}$ can be estimated by:

$$
\frac{1}{2 l+1} \sum_{m} \int d^{2} \hat{\mathbf{n}}|W(\hat{\mathbf{n}})|^{2} Y_{l m}(\hat{\mathbf{n}}) Y_{l+2, m}^{*}(\hat{\mathbf{n}}) .
$$

The value as high as unity indicates that most of Gaussian CMB power is leaked into the fictitious correlation, while a zero value indicates no leakage. We have estimated $D_{l}^{i}$ leakages for the WMAP team's KQ75 and KQ85 mask and show them in Table 
Table A1. D3 Leakage

\begin{tabular}{c|cccc}
\hline$l$ & 10 & 20 & 100 & 200 \\
\hline KQ75 & 0.18 & 0.18 & 0.17 & 0.17 \\
KQ85 & 0.12 & 0.12 & 0.12 & 0.12 \\
\hline
\end{tabular}

A1. We find $D_{l}^{3}$ leakage is as high as $\sim 20 \%$ and other leakgages are $\sim 2 \%$, which are all greater than the expected correlations, given Alfvén wave parameters in Table 1. Therefore, we find CMB maps, which requires the KQ75 or KQ 85 mask, are not suitable for estimation of correlations induced by cosmological Alfvén waves. 